Since publication of their article, Dr. Domchek reports having received an honorarium from Clovis Oncology. No further potential conflict of interest relevant to this letter was reported.

1. Kümmel S, Jackisch C, Müller V, Schneeweiss A, Klawitter S, Lux MP. Can contemporary trials in HER2-negative metastatic breast cancer (mBC) detect overall survival (OS) benefit? Ann Oncol 2017;28:Suppl 5:267P. abstract.

2. Robson M, Ruddy KJ, Im SA, et al. OlympiAD: health-related quality of life (HRQoL) in patients with HER2-negative metastatic breast cancer $(\mathrm{mBC})$ and a germline BRCA mutation (gBRCAm) receiving olaparib monotherapy versus standard single-agent chemotherapy treatment of physician's choice (TPC). Ann Oncol 2017;28:Suppl 5:290P. abstract.

3. Cortazar P, Justice R, Johnson J, Sridhara R, Keegan P, Pazdur R. US Food and Drug Administration approval overview in metastatic breast cancer. J Clin Oncol 2012;30:1705-11.

DOI: $10.1056 / N E J M c 1711644$

\title{
Declining Risk of Sudden Death in Heart Failure
}

TO THE EDITOR: Shen et al. (July 6 issue) ${ }^{1}$ report declining rates of sudden death among patients with heart failure and reduced ejection fraction who were enrolled in trials spanning the period from 1995 through 2014. This message is one of hope,$^{2}$ contrasting with a traditionally more pessimistic narrative. ${ }^{3}$

However, why use simple linear regression? A spline term may better fit the data in Figure 1 (available with the full text of the article at NEJM.org). The patients in the control groups of the BEST (Beta-Blocker Evaluation of Survival) trial, RALES (Randomized Aldactone Evaluation Study), and MERIT-HF (Metoprolol CR/XL Randomised Intervention Trial in Congestive Heart Failure) appear on inspection to be outliers; very few of these patients were receiving beta-blockers. By using a suitable spline, we can envisage a steep decline in the rate of sudden death through the mid-1990s and a flatter trend after MERIT-HF, when beta-blockers became standard treatment. Evidence supporting the use of implantable cardioverter-defibrillators (ICDs) for primary prevention in patients with heart failure with reduced ejection fraction accrued thereafter. ${ }^{4}$

Selection bias is another concern. The proportion of patients with baseline ICDs who were excluded from the trials in the analysis by Shen et al. increased over time, consistent with the trend in clinical use. ${ }^{5}$ None of the patients in the early trials had an ICD at baseline; the rate of ICD use at baseline was 2.7\% in CORONA (Controlled Rosuvastatin Multinational Trial in Heart Failure), 6.4\% in GISSI-HF (Gruppo Italiano per lo Studio della Sopravvivenza nell'Insufficienza Cardiaca Heart Failure Trial), 13\% in EMPHASIS-HF (Eplerenone in Mild Patients Hospitalization and Survival Study in Heart Failure), and approximately 15\% in the PARADIGM-HF trial (Prospective Comparison of ARNI with ACEI to Determine
Impact on Global Mortality and Morbidity in Heart Failure). Indeed, after the exclusion of patients with ICDs, the sample for analysis appeared to be healthier over time (e.g., $29.0 \%$ of the patients in RALES had New York Heart Association [NYHA] functional class IV heart failure with reduced ejection fraction vs. $0.8 \%$ in the PARADIGMHF trial) (Table S4 in the Supplementary Appendix of the article, available at NEJM.org).

Cian P. McCarthy, M.B., B.Ch., B.A.O.

Massachusetts General Hospital

Boston, MA

Killian J. McCarthy, M.B., B.Ch., B.A.O.

Beth Israel Deaconess Medical Center

Boston, MA

John W. McEvoy, M.B., B.Ch., M.H.S.

Johns Hopkins University School of Medicine

Baltimore, MD

jmcevoyl@jhmi.edu

No potential conflict of interest relevant to this letter was reported.

1. Shen L, Jhund PS, Petrie MC, et al. Declining risk of sudden death in heart failure. N Engl J Med 2017;377:41-51.

2. Cleland JGF, Pellicori P, Clark AL, Petrie MC. Time to take the failure out of heart failure: the importance of optimism. JACC Heart Fail 2017;5:538-40.

3. Packer M. Angiotensin neprilysin inhibition for patients with heart failure: what if sacubitril/valsartan were a treatment for cancer? JAMA Cardiol 2016;1:971-2.

4. Moss AJ, Zareba W, Hall WJ, et al. Prophylactic implantation of a defibrillator in patients with myocardial infarction and reduced ejection fraction. N Engl J Med 2002;346:877-83.

5. Schmidt M, Pedersen SB, Farkas DK, et al. Thirteen-year nationwide trends in use of implantable cardioverter-defibrillators and subsequent long-term survival. Heart Rhythm 2015;12: 2018-27.

DOI: $10.1056 / N E J M c 1711901$

TO THE EDITOR: Shen et al. describe a $44 \%$ reduction in the rate of sudden death among patients with heart failure and reduced ejection fraction who were enrolled in 12 trials conducted during the period from 1995 through 2014. The authors 
associate this significant reduction with the sequential introduction of efficacious drugs.

However, patients who were receiving digitalis had a consistently increased incidence of sudden death (Table S5 in the Supplementary Appendix of the article). In a systematic review, digoxin was associated with an increase of $14 \%$ in the risk of death from any cause. ${ }^{1}$ In the ROCKET-AF trial (Rivaroxaban Once Daily Oral Direct Factor Xa Inhibition Compared with Vitamin K Antagonism for Prevention of Stroke and Embolism Trial in Atrial Fibrillation), the use of digoxin was associated with an increase of $33 \%$ in the rate of sudden death. ${ }^{2}$ Therefore, the reduction in the rate of sudden death is also potentially attributable to the decline in the administration of digoxin. In the BEST trial, which was conducted from 1995 through 1998, digoxin was administered in $92 \%$ of the patients, whereas in the PARADIGM-HF trial, which had a run-in period from the end of 2009 through 2012, the drug was listed among treatments at the time of randomization in only $30 \%$ of the patients.

In the United States, there was a relative reduction of $91 \%$ in the rate of prescription of digitalis among patients with heart failure during the period from 1997 through 2012. ${ }^{3}$ This reduction probably contributed to the decline in the rate of sudden death among patients with heart failure and reduced ejection fraction.

\section{Deborah Cosmi, M.D. \\ Ospedale di Gubbio-Gualdo Tadino \\ Gubbio, Italy \\ deborahcosmi@yahoo.it \\ Beatrice Mariottoni, M.B. \\ Franco Cosmi, M.D. \\ Ospedale di Cortona \\ Cortona, Italy}

No potential conflict of interest relevant to this letter was reported.

1. Vamos M, Erath JW, Hohnloser SH. Digoxin-associated mortality: a systematic review and meta-analysis of the literature. Eur Heart J 2015;36:1831-8.

2. Washam JB, Stevens SR, Lokhnygina Y, et al. Digoxin use in patients with atrial fibrillation and adverse cardiovascular outcomes: a retrospective analysis of the Rivaroxaban Once Daily Oral Direct Factor Xa Inhibition Compared with Vitamin K Antagonism for Prevention of Stroke and Embolism Trial in Atrial Fibrillation (ROCKET AF). Lancet 2015;385:2363-70.

3. Goldberger ZD, Alexander GC. Digitalis use in contemporary clinical practice: refitting the foxglove. JAMA Intern Med 2014;174:151-4.

DOI: $10.1056 /$ NEJMc1711901
TO THE EDITOR: Shen et al. report that the risk of sudden death among patients with heart failure and reduced ejection fraction has declined substantially over the past two decades. They attribute this reduction to the widespread use of guideline-based medical therapies, and these therapies may possibly reduce the need for an ICD.

Risk assessment tools to best identify persons who are likely to benefit from ICDs are essential. Thus, it would be interesting to know how well the Seattle Heart Failure Model ${ }^{1}$ and the Seattle Proportional Risk $\mathrm{Model}^{2}$ for sudden death would perform in the populations from both the earlier and the more recent trials assessed by Shen et al. These models were constructed with the use of data from the Prospective Randomized Amlodipine Survival Evaluation (PRAISE) and several other cohorts from the early 1990s to the early 2000s. Thus, we hypothesize that these models may require modifications to adjust for the low incidence of sudden death observed in recent trials, although these models recently were shown to perform fairly well in a nationwide registry of patients with ICDs and heart failure. ${ }^{3}$

Mitsuaki Sawano, M.D.

Shun Kohsaka, M.D.

Keiichi Fukuda, M.D., Ph.D.

Keio University School of Medicine

Tokyo, Japan

mitsuakisawano@keio.jp

No potential conflict of interest relevant to this letter was reported.

1. Levy WC, Mozaffarian D, Linker DT, et al. The Seattle Heart Failure Model: prediction of survival in heart failure. Circulation 2006;113:1424-33.

2. Shadman R, Poole JE, Dardas TF, et al. A novel method to predict the proportional risk of sudden cardiac death in heart failure: derivation of the Seattle Proportional Risk Model. Heart Rhythm 2015;12:2069-77.

3. Bilchick KC, Wang Y, Cheng A, et al. Seattle Heart Failure and Proportional Risk Models predict benefit from implantable cardioverter-defibrillators. J Am Coll Cardiol 2017;69:2606-18.

DOI: 10.1056/NEJMc1711901

THE AUTHORS REPLY: McCarthy et al. ask whether a spline function gives a better fit than simple linear regression for the trend we found in the risk of sudden death over time. Accordingly, we examined the trend using a spline model with the knot set at the time suggested by McCarthy and colleagues. However, the fit was no better than with the linear model $(\mathrm{P}=0.10$ by the $\log$ likelihood test). 
McCarthy et al. raise a second point about selection bias due to exclusion of patients with an ICD. However, the proportion of these patients was small and unlikely to account for our findings. The "healthier" profile of patients in later years was accounted for by adjustment for NYHA functional class, along with a number of other confounding variables, including age, sex, left ventricular ejection fraction, cause of ischemia, previous myocardial infarction, history of hypertension, and diabetes.

Cosmi et al. suggest that the use of digoxin may increase the risk of sudden death among patients with heart failure and that reducing the use of this treatment might have contributed to the trend we observed. However, this purported risk of digoxin has been suggested only by observational analyses that are confounded by indication and in other ways. The only large, prospective, randomized, placebo-controlled trial of digoxin in patients with heart failure and reduced ejection fraction did not show an increase in the rate of death (or sudden death) or in hospital admissions for ventricular arrhythmias or cardiac arrest. ${ }^{1}$

We agree with Sawano and colleagues that it would be of interest to develop better tools to identify patients who are at risk for sudden death (and in whom ICDs might be of most benefit).
We have evaluated the Seattle Heart Failure Model, ${ }^{2}$ the Seattle Proportional Risk Model, ${ }^{3}$ and newer models in more contemporary trial data sets. Briefly, the Seattle Heart Failure Model was less discriminating at predicting sudden death, death from pump failure, and death from any cause in these more recent data sets than in older data sets. Likewise, the Seattle Proportional Risk Model showed poor discrimination in contemporary cohorts, since both models assessed most patients as being appropriate candidates for an ICD.

Li Shen, M.B., Ch.B.

Pardeep S. Jhund, M.B., Ch.B., Ph.D.

John J.V. McMurray, M.D.

University of Glasgow

Glasgow, United Kingdom

john.mcmurray@glasgow.ac.uk

Since publication of their article, the authors report no further potential conflict of interest.

1. The Digitalis Investigation Group. The effect of digoxin on mortality and morbidity in patients with heart failure. N Engl J Med 1997;336:525-33.

2. Mozaffarian D, Anker SD, Anand I, et al. Prediction of mode of death in heart failure: the Seattle Heart Failure Model. Circulation 2007;116:392-8.

3. Shadman R, Poole JE, Dardas TF, et al. A novel method to predict the proportional risk of sudden cardiac death in heart failure: derivation of the Seattle Proportional Risk Model. Heart Rhythm 2015;12:2069-77.

DOI: 10.1056/NEJMc1711901

\section{Post-Traumatic Stress Disorder}

TO THE EDITOR: The review by Shalev et al. (June 22 issue) ${ }^{1}$ highlights the need for new effective treatments for post-traumatic stress disorder (PTSD). One treatment that Shalev et al. did not describe is animal-assisted therapy. Since my first proposal and description of a case involving such therapy, ${ }^{2}$ it has come into widespread use for PTSD. ${ }^{3}$ Hundreds of organizations and chapters are now providing therapy animals for patients. More formal study is needed. ${ }^{4}$ A randomized, controlled trial of animal-assisted therapy for PTSD is currently under way (ClinicalTrials.gov number, NCT02039843). Not just dogs but horses and parrots are being used to treat patients with PTSD, including as an aid in suicide prevention (although such descriptions of cases have been anecdotal). Unlike currently used medications, cognitive behavioral therapy, or experimental treatments with unapproved medications, the use of magnetic fields, or interventions requiring close timing to the inciting traumatic event, therapy with animals can be used in the acute or chronic phase of PTSD and for patients who have had sexual or war trauma. ${ }^{4}$ Such therapy has excellent adherence, along with a favorable sideeffect profile.

\section{Eric L. Altschuler, M.D., Ph.D.}

Metropolitan Hospital Center

New York, NY

altschue@nychhc.org

No potential conflict of interest relevant to this letter was reported.

1. Shalev A, Liberzon I, Marmar C. Post-traumatic stress disorder. N Engl J Med 2017;376:2459-69.

2. Altschuler EL. Pet-facilitated therapy for posttraumatic stress disorder. Ann Clin Psychiatry 1999;11:29-30.

3. Pandzic I. Animal-assisted therapy and PTSD. White paper. 